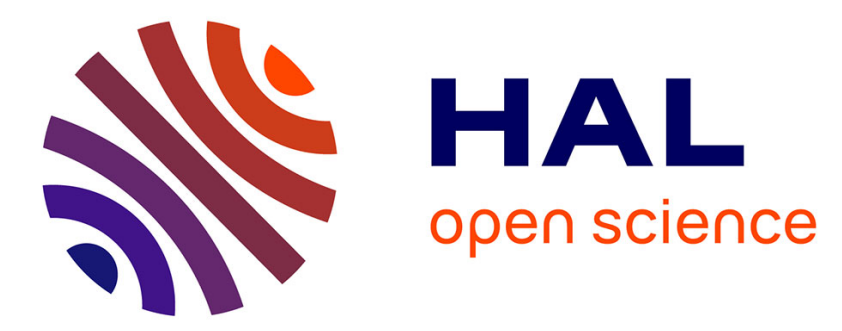

\title{
Parameter and state estimation for a class of neural mass models
}

\author{
Romain Postoyan, Michelle Chong, Dragan Nesic, Levin Kuhlmann
}

\section{To cite this version:}

Romain Postoyan, Michelle Chong, Dragan Nesic, Levin Kuhlmann. Parameter and state estimation for a class of neural mass models. 51st IEEE Conference on Decision and Control, CDC 2012, Dec 2012, Maui, Hawaii, United States. pp.CDROM. hal-00753114

\section{HAL Id: hal-00753114 https://hal.science/hal-00753114}

Submitted on 23 Dec 2012

HAL is a multi-disciplinary open access archive for the deposit and dissemination of scientific research documents, whether they are published or not. The documents may come from teaching and research institutions in France or abroad, or from public or private research centers.
L'archive ouverte pluridisciplinaire HAL, est destinée au dépôt et à la diffusion de documents scientifiques de niveau recherche, publiés ou non, émanant des établissements d'enseignement et de recherche français ou étrangers, des laboratoires publics ou privés. 


\title{
Parameter and state estimation for a class of neural mass models
}

\author{
Romain Postoyan, Michelle Chong, Dragan Nešić and Levin Kuhlmann.
}

\begin{abstract}
We present an adaptive observer which asymptotically reconstructs the parameters and states of a model of interconnected cortical columns. Our study is motivated by the fact that the considered model is able to realistically reproduce patterns seen on (intracranial) electroencephalograms (EEG) by varying its parameters. Therefore, by estimating its parameters and states, we could gain a better understanding of the mechanisms underlying neurological phenomena such as seizures, which might lead to the prediction of the onsets of epileptic seizures. Simulations are performed to illustrate our results.
\end{abstract}

\section{INTRODUCTION}

A long standing quest in brain research is to understand the underlying physiological mechanisms involved in a neurological phenomenon by using measurable signals such as the electroencephalogram (EEG). One way in which this challenge can be undertaken is via estimating the states and parameters of mathematical models that have been devised to capture various brain phenomena. In particular, the emergence of models which are able to realistically reproduce patterns seen on (intracranial) EEG shows great potential (see [5]). Indeed, if we could estimate the states and parameters of these models online, we would have access to neurophysiologically relevant data which are not observable today. This would allow us to better understand the physiological mechanisms and could potentially lead to the development of new therapeutic treatments of neurological diseases, such as the prediction of the onset of an epileptic seizure for abatement via electrical stimulation or the administration of drugs.

We focus on lumped parameter models developed in [8] and [13] that describes the electrical activity of the cortex at the scale of populations of neurons. These models are also known as neural mass models in the literature [5]. In [8], a single cortical column model was presented which originated from the seminal work of Lopes da Silva [4]. This model is of particular interest for two reasons. Firstly, it is able to generate an output signal which mimics the recorded flash evoked potential in the EEG. Secondly, it can be used as a basic unit that models a specific region of the cortex and these units can then be interconnected to describe the interaction between different cortical regions, see [8] and [13]. For these reasons, a model of $n$ coupled neural populations is proposed in [13] which generalises the coupled

R. Postoyan is with the Université de Lorraine, CRAN, UMR 7039 and the CNRS, CRAN, UMR 7039, France. He is financially supported by the PEPS-CNRS project APSE. romain.postoyan@univ-lorraine.fr

M. Chong, D. Nešić and L. Kuhlmann are with the Department of Electrical and Electronic Engineering, the University of Melbourne, Australia. \{chongms, dnesic, levink\}@unimelb.edu.au cortical column model given in [8]. The authors of [8] and [13] identified regions of the parameter space that produce different types of oscillations seen in the EEG (measured output). These oscillatory patterns are known to be related to various brain activity. Hence, estimating the parameters may give researchers a glimpse into the current state of the brain and provide insights into the spatial aspects of brain activity, such as the spreading of seizure activity in an epileptic brain [13]. Moreover, the estimation of the states might lead to the development of intracranial feedback control strategies to abate seizures, see [11].

Few researchers have proceeded in this direction, where stochastic filters are usually the observers of choice [11] One of the reasons we choose to tackle the problem using deterministic adaptive observers is so that we can provide an analytical proof of the convergence of the estimates.

We observe that the models in [8] and [13] admit the following general structure:

$$
\begin{aligned}
\dot{x}_{0} & =A_{0} x_{0}+\phi_{0}(y) \theta \\
\dot{x}_{1} & =A_{1} x_{1}+\phi_{1}\left(x_{0}, u\right) \theta \\
y & =C_{1} x_{1},
\end{aligned}
$$

where $x_{0} \in \mathbb{R}^{n_{0}}, x_{1} \in \mathbb{R}^{n_{1}}$ are the states, $\theta \in \mathbb{R}^{p}$ is a vector of constant and unknown parameters, $y \in \mathbb{R}^{n_{y}}$ is the measurement and $u \in \mathbb{R}^{n_{u}}$ is the input.

To the best of our knowledge, there is currently no adaptive observer in the literature that can be used to estimate the parameters and the states of system (1). Indeed, most nonlinear adaptive observers apply to systems for which the nonlinearities depend on known quantities, see [15] for instance. This is not the case for system (1) since $\phi_{1}$ depends on $x_{0}$ which is not measured. Few designs have been proposed for systems with state-dependent nonlinear terms, see [12] and the references therein. A notable exception is the work in [6] which focuses on a class of systems that is very similar to (1). While the nonlinearities in (1) satisfy some of the conditions stated in [6], the problem arises in the linear part which is not of the same form as in [6]. As a consequence, we need to modify the observer and the technical proof in [6]. This study extends our previous works [2] and [3] on the state estimation of this class of neural mass models.

The paper is organised as follows: The considered class of neural mass models is presented in Section II. We formulate the problem in Section III. The adaptive observer is presented in Section IV and simulations results are provided in Section V. Section VI concludes the paper. All technical lemmas and proofs are provided in the Appendix. 


\section{Definition and Notation}

- A vector $\left[\begin{array}{l}a \\ b\end{array}\right]$ is denoted $(a, b)$, for all $a \in \mathbb{R}^{n_{a}}, b \in$ $\mathbb{R}^{n_{b}}$.

- A block diagonal matrix with square matrices $A_{i} \in \mathbb{R}^{n_{i} \times n_{i}}$ for $i \in\{1, \ldots, n\}$ is denoted as $\operatorname{diag}\left(A_{1}, \ldots, A_{n}\right)$.

- The $n$-by- $n$ identity matrix is denoted by $\mathbb{I}_{n}$, where integer $n>0$.

- The set of positive real scalars is denoted by $\mathbb{R}_{\geq 0}$ and the set of strictly positive real scalars is denoted $\mathbb{R}_{>0}$.

- A continuous function $\beta:[0, \infty) \times[0, \infty) \rightarrow[0, \infty)$ is said to be a class $\mathcal{K} \mathcal{L}$ function, if, for each fixed $s$, the mapping $\beta(r, s)$ is strictly increasing with respect to $r, \beta(0, s)=0$, and for each fixed $r$, the mapping $\beta(r, s)$ is decreasing with respect to $s$ and $\beta(r, s) \rightarrow 0$ as $s \rightarrow \infty$.

\section{DESCRIPTION OF THE MODELS}

\section{A. A single cortical column model}

We consider a model of a single cortical column presented in Section 2.1 in [8] that describes the functional relationship between neuronal populations. This model captures the interactions between the pyramidal neurons, the excitatory and inhibitory interneurons in the cortex and is able to reproduce the oscillatory patterns seen in the EEG (measured output) by tuning the synaptic gains of the excitatory and inhibitory interneurons (parameters). To write the model in state space form (1), we take the states to be ${ }^{1} x_{0}=\left(x_{01}, x_{02}\right) \in \mathbb{R}^{2}$ and $x_{1}=\left(x_{11}, \ldots, x_{14}\right) \in \mathbb{R}^{4}$, where $x_{01}, x_{11}, x_{13}$ are membrane potential contributions of the pyramidal neurons, the excitatory and inhibitory interneurons respectively, and $x_{02}, x_{12}, x_{14}$ are their respective derivatives. The measured output (EEG) is $y \in \mathbb{R}, u \in \mathbb{R}$ is the excitatory input from neighbouring columns which is assumed to be known and $\theta=\left(\theta_{A}, \theta_{B}\right) \in \Theta \subseteq \mathbb{R}^{2}$ is a vector of unknown parameters where $\theta_{A}$ and $\theta_{B}$ represent the synaptic gains of the excitatory and inhibitory neuronal populations respectively. The matrices in (1) are defined as:

$$
\begin{aligned}
& C_{1}=\left(\begin{array}{cccc}
1 & 0 & -1 & 0
\end{array}\right), A_{0}=A_{a}, A_{1}=\operatorname{diag}\left(A_{a}, A_{b}\right), \\
& \text { where } A_{a}=\left(\begin{array}{cc}
0 & 1 \\
-a^{2} & -2 a
\end{array}\right), \quad A_{b}=\left(\begin{array}{cc}
0 & 1 \\
-b^{2} & -2 b
\end{array}\right) .
\end{aligned}
$$

The parameters $a, b \in \mathbb{R}_{>0}$ are assumed to be known. It has to be noted that the matrices $A_{0}$ and $A_{1}$ are Hurwitz. The nonlinear terms in (1) are given by:

$$
\begin{aligned}
\phi_{0}(y) & =\left(\begin{array}{cc}
0 & 0 \\
a S(y) & 0
\end{array}\right), \\
0 & \left(\begin{array}{cc}
a c_{2} S\left(c_{1} x_{01}\right)+a u & 0 \\
0 & 0 \\
0 & b c_{4} S\left(c_{3} x_{01}\right)
\end{array}\right) .
\end{aligned}
$$

\footnotetext{
${ }^{1}$ According to the notation of [8], $x_{0}=\left(y_{0}, y_{3}\right)$ and $x_{1}=$ $\left(y_{1}, y_{4}, y_{2}, y_{5}\right)$.
}

The parameters $c_{1}, c_{2}, c_{3}, c_{4} \in \mathbb{R}_{\geq 0}$ are assumed to be known parameters and $S$ denotes the sigmoid function, for $v \in \mathbb{R}$ : $S(v)=\frac{2 e_{0}}{1+e^{r\left(v_{0}-v\right)}}$ with known constants $e_{0}, v_{0}, r \in \mathbb{R}_{>0}$. For a detailed description about the model and its parameters, see [8].

\section{B. Interconnected single cortical column models}

We now consider a model of $n$ inteconnected cortical columns presented in [13]. This model is obtained by interconnecting $n$ of the single cortical column models described in Section II-A, in the manner shown in Figure 1. Physiologically, the interactions between cortical columns are the firing rates of each column $S\left(y_{i}\right)$. Hence, a physiological interpretation of Figure 1 would include the sigmoid block $S$ as part of the single column model.

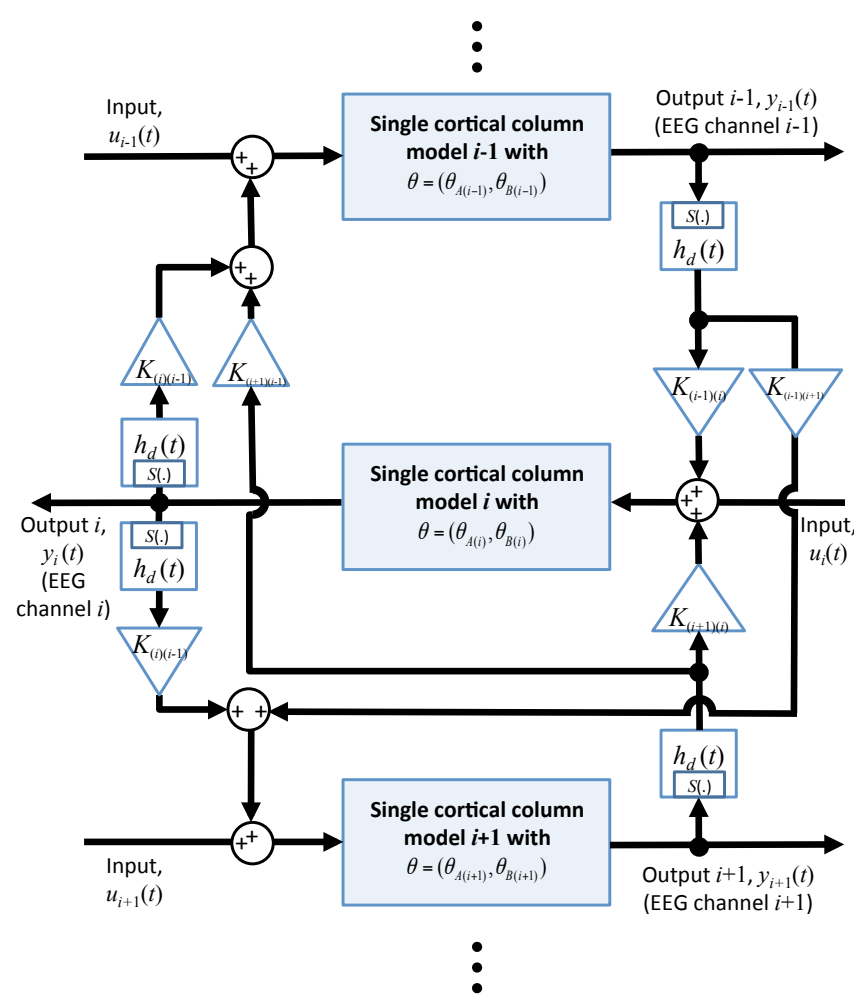

Fig. 1. $n$-interconnected models, where $i \in\{2, \ldots, n-1\}$.

The study of the interconnected models has the potential of gaining a better understanding of the interactions between different regions, whereby each model represents a part of the cortex [13]. The strength of their interaction is captured by the linear gains $K_{j i}$, for $i, j \in\{1, \ldots, n\}$ and $i \neq j$. The time delay of interaction between these regions is described by:

$$
h_{d}(t)=\theta_{A} a_{d} t \exp \left(-a_{d} t\right), \quad \forall t \geq 0,
$$

where $\theta_{A}, a_{d}$ are positive constants defined in Table 1 of [13].

We assume that the synaptic gains of the populations $\theta_{A i}$ and $\theta_{B i}$ are unknown and the coupling gains $K_{j i}$ for $i, j \in$ $\{1, \ldots, n\}$ and $i \neq j$ are known. The interconnected models 
can then be written in the form of (1) by taking the states in (1) to be ${ }^{2} x_{0}=\left(x_{01}^{1}, x_{02}^{1}, x_{03}^{1}, x_{04}^{1}, \ldots, x_{01}^{n}, x_{02}^{n}, x_{03}^{n}, x_{04}^{n}\right)$ and $x_{1}=\left(x_{11}^{1}, x_{12}^{1}, x_{13}^{1}, x_{14}^{1}, \ldots, x_{11}^{n}, x_{12}^{n}, x_{13}^{n}, x_{14}^{n}\right)$, where integer $n>1$. The input is $u=\left(u_{1}, \ldots, u_{n}\right)$ and measurement $y=\left(y_{1}, \ldots, y_{n}\right)$. The matrices are:

$$
\begin{aligned}
& A_{0}=\operatorname{diag}\left(A_{01}, \ldots, A_{0 n}\right), \\
& A_{1}=\operatorname{diag}\left(A_{11}, \ldots, A_{1 n}\right), \\
& C_{1}=\left(\begin{array}{ccc}
C_{11} & \ldots & C_{1 n}
\end{array}\right),
\end{aligned}
$$

where $C_{1 i}=\left(\begin{array}{cccc}1 & 0 & -1 & 0\end{array}\right), A_{0 i}=\operatorname{diag}\left(A_{a}, A_{d}\right)$ and $A_{1 i}=\operatorname{diag}\left(A_{a}, A_{b}\right)$ for $i \in\{1, \ldots, n\}, A_{a}$ and $A_{b}$ are as defined in (2) and $A_{d}=\left(\begin{array}{cc}0 & 1 \\ -a_{d}^{2} & -2 a_{d}\end{array}\right)$. The nonlinear terms in (1) are:

$$
\begin{aligned}
\phi_{0}(y) & =\left[\begin{array}{lll}
\phi_{01} & \ldots & \phi_{0 n}
\end{array}\right], \\
\phi_{1}\left(x_{0}, u\right) & =\left[\begin{array}{lll}
\phi_{11} & \ldots & \phi_{1 n}
\end{array}\right],
\end{aligned}
$$

where for $i \in\{1, \ldots, n\}$,

$$
\begin{aligned}
\phi_{0 i} & =\left(\begin{array}{cc}
0 & 0 \\
a S\left(y_{i}\right) & 0 \\
0 & 0 \\
a_{d} S\left(y_{i}\right) & 0
\end{array}\right), \\
0 & \\
\phi_{1 i} & =\left(\begin{array}{cc}
a u_{i}+a c_{2} S\left(c_{1} x_{01}^{i}\right) & \\
+\sum_{j \in\{1, \ldots, n\}, j \neq i} K_{j i} x_{03}^{j} & 0 \\
0 & 0 \\
0 & b c_{4} S\left(c_{3} x_{01}^{i}\right)
\end{array}\right) .
\end{aligned}
$$

\section{PROBLEM FORMULATION AND ASSUMPTIONS}

For ease of notation, we write (1) in the following form:

$$
\begin{aligned}
& \dot{x}=A x+\phi(y, u, x) \theta \\
& y=C x,
\end{aligned}
$$

where $x=\left(x_{0}, x_{1}\right), A=\operatorname{diag}\left(A_{0}, A_{1}\right), C=\left(0, C_{1}\right)$ and $\phi=\left(\phi_{0}, \phi_{1}\right)$. The nonlinear terms $\phi_{0}: \mathbb{R} \rightarrow \mathbb{R}^{n_{0}} \times \mathbb{R}^{p}$ and $\phi_{1}: \mathbb{R}^{n_{0}} \times \mathbb{R}^{n_{u}} \rightarrow \mathbb{R}^{n_{1}} \times \mathbb{R}^{p}$ are globally Lipschitz and bounded. The matrices $A_{0}$ and $A_{1}$ are Hurwitz.

Our objective is to synthesise an adaptive observer to simultaneously estimate the state $x$ and parameter $\theta$ of (1) from the measured output $y$. We make the following crucial assumptions in the design of the adaptive observer:

Assumption 1: The vector of unknown parameters $\theta$ is constant and is known to reside in a compact set $\Theta$.

This assumption is justified under the circumstances where the parameters are slowly-varying for each type of cortical activity [8]. When a change in activity occurs, an abrupt variation of $\theta$ is presumed which violates Assumption 1 for only a short time as illustrated in simulations in Section V. For the models described in Section II, the plant's parameters $\theta^{\star}$ were identified to lie in a compact set in Sections 3.1 and 3.2 of [8] respectively.

Assumption 2: The input $u$ is known.

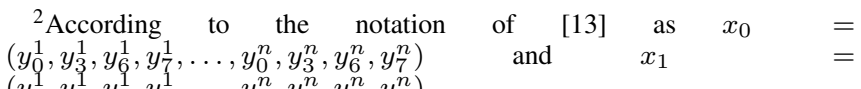

This is a limiting assumption in practice as the input is unknown/unmeasured. A common assumption is to model the input as random white noise, see [8], [13].

Assumption 3: The measured output $y$ is noise-free. This assumption is more justified when electroencephalogram (EEG) recorded with intracranial electrodes as opposed to EEG obtained from electrodes placed on the scalp. We investigate the robustness of our algorithm to measurement noise in simulations in Section $\mathrm{V}$.

\section{MAIN RESULTS}

We consider the following adaptive observer for system (4):

$$
\begin{array}{ll}
\dot{\hat{x}}=A \hat{x}+\phi(y, u, \hat{x}) \hat{\theta}+\Gamma(y-\hat{y}) & \\
\hat{y}=C \hat{x} & \\
\dot{\hat{\theta}}=\bar{\Gamma}(y-\hat{y}) & \text { with } \Upsilon(0)=0 \\
\dot{\Upsilon}=A \Upsilon+\Delta \phi(y, u, \hat{x}) & \text { with } P(0)=P(0)^{T}>0, \\
\dot{P}=d P-d P \Upsilon^{\mathrm{T}} C^{\mathrm{T}} C \Upsilon P &
\end{array}
$$

where $\Gamma=\Delta^{-1} \Upsilon \bar{\Gamma}, \bar{\Gamma}=P \Upsilon^{\mathrm{T}} C^{\mathrm{T}}$ and $\Delta=\operatorname{diag}\left(\mathbb{I}_{n_{0}}, \frac{1}{d} \mathbb{I}_{n_{1}}\right)$ with $d>0$ a design parameter. The vector $\hat{x}$ denotes the estimate of $x$ and $\hat{\theta}$ the estimate of $\theta$. The variable $\Upsilon \in$ $\mathbb{R}^{\left(n_{0}+n_{1}\right) \times p}$ is initialized to $\Upsilon(0)=0$ in order to simplify the technical statements. Our result also applies when it is not the case. An essential assumption for our design to work is the condition stated below.

Assumption 4: For any signals $u, y, \hat{x}$ that belong to $\mathcal{L}_{\infty}$, there exist $a_{1}, a_{2} \in \mathbb{R}_{>0}, T \in \mathbb{R}_{>0}$ such that the solution to:

$$
\dot{\Upsilon}=A \Upsilon+\Delta \phi(y, u, \hat{x}) \text { with } \Upsilon(0)=0,
$$

satisfies for all $^{3} t \geq 0$ :

$$
a_{1} \mathbb{I}_{2} \leq \int_{t}^{t+T} \Upsilon^{\mathrm{T}}(\tau) C^{\mathrm{T}} C \Upsilon(\tau) \mathrm{d} \tau \leq a_{2} \mathbb{I}_{2}
$$

The condition (7) is known as the persistency of excitation of the signal $C \Upsilon(t)$ and is a well-known condition in identification and adaptive control literature (see [7]). This is a sufficient condition for the identification of the parameter $\theta$ using the adaptive observer proposed here and it is similar to the condition used in (A4) of [6] and (A3) in [1]. Inequality (7) is hard to verify analytically in general. Nevertheless, we have observed in simulations that this condition is satisfied for the model we consider under the simulation conditions stated in Section V.

The matrix $P$ will be used to construct a Lyapunov function in the proof. It can be verified that $P(t)$ is symmetric and positive definite with lower and upper bound which are independent of $d$, see Lemma 1 in [16].

We are now ready to state the main result which ensures the asymptotic convergence of the estimated variables $(\hat{x}, \hat{\theta})$ to $(x, \theta)$. Its proof is given in the Appendix.

\footnotetext{
${ }^{3}$ A unique solution exists for all time, for all the ordinary differential equations in (4) and (5). By Theorem 3.3 of [10], $\dot{P}=d P-$ $d P \Upsilon^{T} C^{T} C \Upsilon P$ in (5) has a unique solution because its right hand side is locally Lipschitz and piecewise continuous in $t$ and all its solution are in a compact set (by Lemma 1 of [16], $P$ is bounded). The other ODEs in (4) and (5) have unique solutions because the nonlinearity $\phi$ is globally Lipschitz.
} 
Theorem 1: Consider the system (4) and the observer (5) and suppose Assumptions 1-4 are satisfied. Then, there exists $d^{\star} \geq 1$ such that for all $d \geq d^{\star}$, the estimates $(\hat{x}, \hat{\theta})$ asymptotically converge towards $(x, e)$ i.e. for any $d \geq d^{\star}$, there exist $\beta_{d} \in \mathcal{K} \mathcal{L}$ such that for any input $u$ and any initial conditions $P(0)=P(0)^{\mathrm{T}}>0, \tilde{x}(0)$ and $\tilde{\theta}(0)$, the following holds :

$$
|(\tilde{x}(t), \tilde{\theta}(t))| \leq \beta_{d}(|(\tilde{x}(0), \tilde{\theta}(0))|, t) \quad \forall t \geq 0,
$$

where $\tilde{x}:=x-\hat{x}$ and $\tilde{\theta}:=\theta-\hat{\theta}$.

Remark 1: The design parameter $d$ is chosen such that $d \geq d^{\star}$ to obtain (8). An estimate of $d^{\star}$, which we believe is subject to some conservatism is provided in the proof of Theorem 1 (see (13) in the Appendix). Although the expression of $d^{\star}$ depends on $|\theta|$, it is possible to estimate it be taking the maximum over the set $\Theta$, where $\theta$ is known to belong to according to Assumption 1 .

\section{SIMULATIONS}

We have performed simulations for the single cortical column model described in Section II-A with the following initial conditions: $x(0)=(0.6,1,0.6,1,0.6,1), \hat{x}(0)=0$, $\theta=(3.25,22), \hat{\theta}(0)=0, P(0)=\mathbb{I}_{2}, \Upsilon(0)=0$. The other parameters take the value given in [8] and the input is a Gaussian noise of mean 100 and variance $30^{2}$. Figures 2-3 respectively show the state and parameter estimation error when the design parameter $d$ is equal to 2 and 10 . They confirm the convergence properties of the algorithm and show that larger $d$ speeds up the rate of convergence.
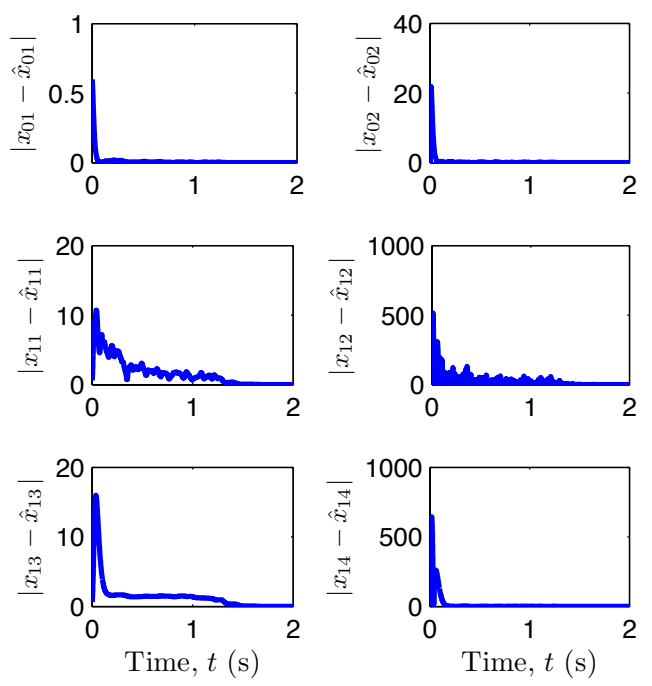

Fig. 2. State estimation error for $d=10$.

As previously mentioned, Assumptions 1 and 3 (i.e parameters are constant and noise-free measurement) are limiting assumptions in practice. In Figure 4, we simulate a change in parameters which might correspond to a change in cortical activity. We see that the estimated parameters still converge to the true values after the transition has occurred. We also consider the scenario where the measured output (EEG) is
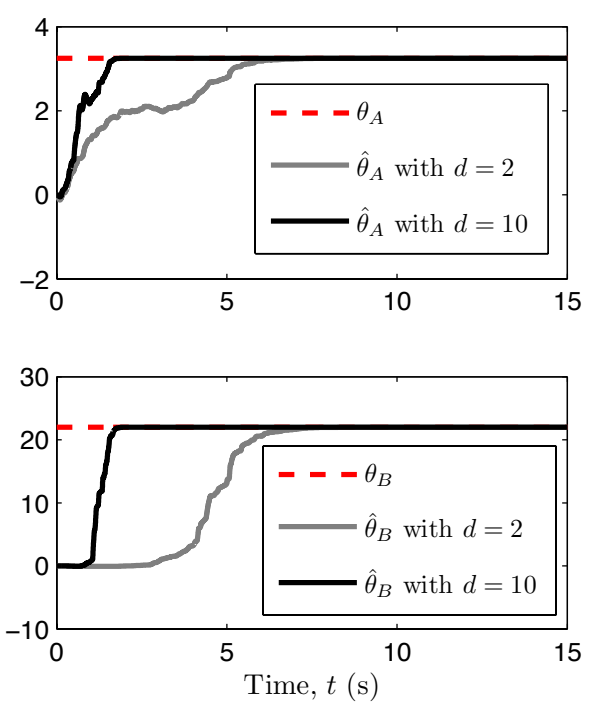

Fig. 3. Parameter estimates for $d=2$ and 10 .

noisy by introducing a random noise that is drawn from a Gaussian distribution of mean 0 and variance $0.4^{2}$, which is approximately $20 \%$ of $y$ at steady state. Figure 5 shows that despite the presence of measurement noise, the adaptive observer still provides estimates that are close to the true values. In fact, the neighbourhood in which the parameter error converges to is smaller with small $d$. This illustrates the tradeoff between fast convergence rate and robustness towards measurement noise.

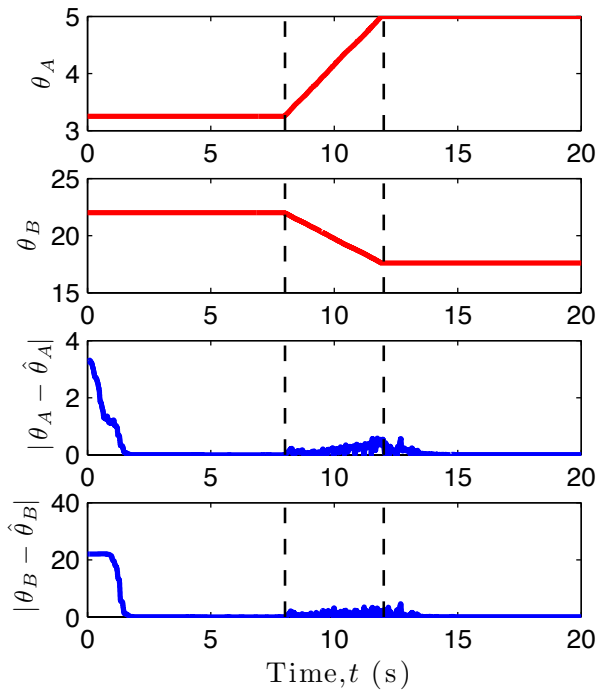

Fig. 4. Parameter estimation error with varying parameters for $d=10$.

\section{CONCLUSION AND FURTHER WORKS}

We have presented an algorithm to provide simultaneous estimates of the states and parameters of a class neural mass models using the electroencephalogram (EEG) as the measured output. This adaptive observer is based on the work in [6], where the class of systems considered are different in the linear part. Consequently, modifications were made 

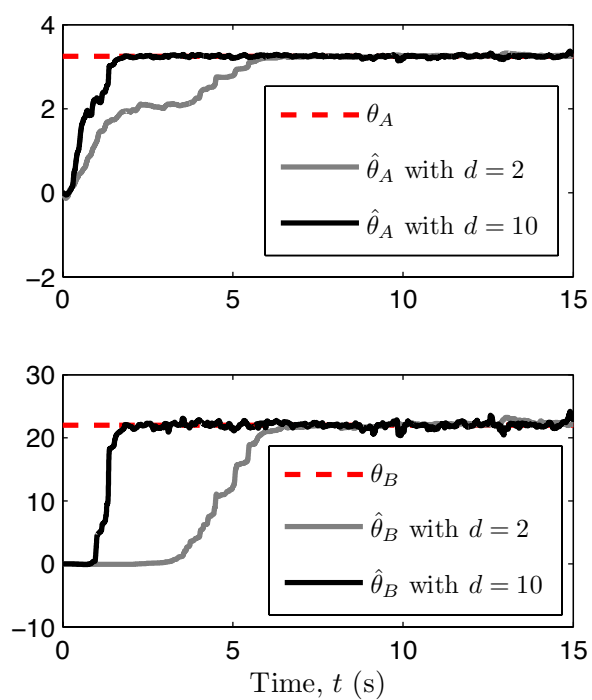

Fig. 5. Parameter estimates with noisy measurement for $d=2$ and 10 .

in the adaptive observer and we provide a technical proof for the asymptotic convergence of parameters and states. Simulations are provided for a single cortical column model by [8] which confirm our results. In the future, we will work on estimating the connectivity gains (see Section II-B), in addition to the synaptic gains $\theta_{A i}$ and $\theta_{B i}, i \in\{1, \ldots, n\}$ of the interconnected model. The adaptive observer presented here does not apply as the nonlinearity $\phi_{1}$ of (1) is no longer bounded as required. Estimating the connectivity gains of interconnected models is of interest as each model serves to model a part of the cortex and the connectivity gain between these models may provide insight into the spatial aspects of the physiological mechanisms involved during a brain phenomenon, such as seizures.

\section{APPENDIX}

\section{A. A technical lemma}

In the proof of Theorem 1, we use that $\Upsilon$ is bounded according to the following lemma.

Lemma 1: Consider system (5). There exist $M \in \mathbb{R}_{>0}$ such that for any $d \geq 1$, any $\mathcal{L}_{\infty}$ signal $\hat{x}, y, u$ the solution of $\dot{\Upsilon}=A \Upsilon+\Delta \phi(y, u, \hat{x})$ with $\Upsilon(0)=0$, satisfies $|\Upsilon(t)| \leq M$ for all $t \geq 0$.

Proof. Note that $|\Delta \phi(y, u, \hat{x})| \leq|\Delta||\phi(y, u, \hat{x})| \leq$ $|\phi(y, u, \hat{x})|$ since $d \geq 1$. Moreover, it can be verified that $\phi$ is upper bounded by a constant (independent of $d$ ) in view of Section II. As a consequence, we can directly conclude that $|\Upsilon(t)|$ can be bounded by a constant $M$ independent of $d$ for all time since the matrix $A$ is Hurwitz and of the initial condition $\Upsilon(0)$ as it was chosen to be $\Upsilon(0)=0$ in the algorithm (5).

\section{B. Proof of Theorem 1}

We first consider the system in $\tilde{x}$ coordinate. According to (4) and (5), we have

$$
\begin{aligned}
\dot{\tilde{x}}= & A \tilde{x}+(\phi(y, u, x)-\phi(y, u, \hat{x})) \theta+\phi(y, u, \hat{x}) \tilde{\theta} \\
& -\Gamma(y-\hat{y}) .
\end{aligned}
$$

We scale the error $\tilde{x}$ as such $\bar{x}:=\Delta \tilde{x}$. Noting that $\Delta A \Delta^{-1}=$ A:

$$
\begin{aligned}
\dot{\bar{x}}= & A \bar{x}+\Delta(\phi(y, u, x)-\phi(y, u, \hat{x})) \theta+\Delta \phi(y, u, \hat{x}) \tilde{\theta} \\
& -\Delta \Gamma(y-\hat{y}) .
\end{aligned}
$$

We now proceed to a dynamical change of coordinates by introducing $\eta$ as follows which was as proposed in [14]:

$$
\eta:=\bar{x}-\Upsilon \tilde{\theta} .
$$

Noting that $C \Delta^{-1}=d C$, the dynamics of variables $\eta$ and $\tilde{\theta}$ are given by:

$$
\left\{\begin{array}{l}
\dot{\eta}=A \eta+\Delta(\phi(y, u, x)-\phi(y, u, \hat{x})) \theta \\
\dot{\tilde{\theta}}=-d P \Upsilon^{\mathrm{T}} C^{\mathrm{T}} C(\eta+\Upsilon \tilde{\theta}) .
\end{array}\right.
$$

We note that, contrary to [14], we have not obtained a nice cascade system since the $\eta$-system also depends on $x$ and $\hat{x}$. In the following, we will invoke small-gain type arguments to conclude the stability of system (10). We consider the Lyapunov-type functions $V_{1}(\eta)=\eta^{\mathrm{T}} S \eta$ and $V_{2}(\tilde{\theta}, P)=$ $\tilde{\theta}^{\mathrm{T}} P^{-1} \tilde{\theta}$ where $S$ is a real symmetric positive definitive matrix such that $A^{\mathrm{T}} S+S A=-\mathbb{I}$ (such a matrix always exists according to Theorem 4.6 in [10] since $A$ is Hurwitz). It can be noted that $V_{2}$ satisfies $\lambda_{1}|\tilde{\theta}|^{2} \leq V_{2}(\tilde{\theta}, P) \leq \lambda_{2}|\tilde{\theta}|^{2}$ where $\lambda_{1}, \lambda_{2}$ are independent of $d$ for $d \geq 1$ according to [16, Lemma 1], which will be useful for our purpose. We first consider $V_{1}$. Along solutions to (10), we have:

$$
\dot{V}_{1}(t)=-|\eta|^{2}+2 \eta^{\mathrm{T}} S \Delta(\phi(y, u, x)-\phi(y, u, \hat{x})) \theta .
$$

Noting that $\phi(x, y, u)=\left(\phi_{0}(y), \phi_{1}\left(x_{0}, u\right)\right)$, so $\phi(x, y, u)-$ $\hat{\phi}(\hat{x}, y, u)=\left(0, \phi_{1}\left(x_{0}, u\right)-\hat{\phi}_{1}\left(\hat{x}_{0}, u\right)\right)$. As a consequence,

$$
\Delta(\phi(y, u, x)-\hat{\phi}(y, u, \hat{x}))=\left(0, \frac{1}{d}\left(\phi_{1}\left(x_{0}, u\right)-\hat{\phi}_{1}\left(\hat{x}_{0}, u\right)\right)\right)
$$

from which we deduce in (11):

$$
\begin{aligned}
\dot{V}_{1}(t) & \leq-|\eta|^{2}+2|\eta||S||\theta|\left|\Delta\left(\phi\left(x_{0}, u\right)-\phi\left(\hat{x}_{0}, u\right)\right)\right| \\
& \leq-|\eta|^{2}+2|\eta||S||\theta|\left(\frac{1}{d}\left|\phi_{1}\left(x_{0}, u\right)-\phi_{1}\left(\hat{x}_{0}, u\right)\right|\right) .
\end{aligned}
$$

By assumption, $\phi_{1}$ is globally Lipschitz with a constant that we denote $L_{1}>0$, thus $\left|\phi_{1}\left(x_{0}, u\right)-\phi_{1}\left(\hat{x}_{0}, u\right)\right| \leq L_{1}\left|\tilde{x}_{0}\right|$. Note also that the scaling induced by the matrix $\Delta$ does not affect the $\tilde{x}_{0}$ part of the estimation error $\tilde{x}$, so that we have $\tilde{x}_{0}=\bar{x}_{0}$. As a consequence,

$$
\begin{aligned}
\dot{V}_{1}(t) & \leq-|\eta|^{2}+\frac{2}{d}|\eta||S||\theta| L_{1}\left|\tilde{x}_{0}\right| \\
& \leq-|\eta|^{2}+\frac{2}{d}|\eta||S||\theta| L_{1}(|\eta|+|\Upsilon \tilde{\theta}|) \\
& =\left(-1+\frac{2}{d}|S||\theta| L_{1}\right)|\eta|^{2}+\frac{2}{d}|\eta||S||\theta| L_{1}|\Upsilon \tilde{\theta}| .
\end{aligned}
$$

We define

$$
d^{\star}:=\max \left\{1,4 L_{1}|\theta||S|\right\} .
$$

Choose $d$ such that $d \geq d^{\star}$, so that $-1+\frac{2}{d}|S||\theta| L_{1}<-\frac{1}{2}$, in that way: $\dot{V}_{1}(t) \leq-\frac{1}{2}|\eta|^{2}+\frac{2}{d}|S| L_{1}|\theta||\eta||\Upsilon \tilde{\theta}|$. According to 
Lemma 1 , we always have $|\Upsilon| \leq M$, therefore: $\dot{V}_{1}(t) \leq$ $-\frac{1}{2}|\eta|^{2}+\frac{2}{d}|S||\theta| L_{1} M|\eta||\tilde{\theta}|$, from which we deduce, by invoking the fact that $S$ and $P^{-1}$ are symmetric positive definite with lower and upper bounds independent of $d$ (see [16, Lemma 1]), that there exists $\sigma_{1}, \sigma_{2} \in \mathbb{R}_{>0}$ independent of $d$ such that:

$$
\begin{aligned}
\dot{V}_{1}(t) & \leq-\sigma_{1} V_{1}+\frac{1}{d} \sigma_{2} \sqrt{V_{1}} \sqrt{V_{2}} \\
& =-\frac{\sigma_{1}}{2} V_{1}-\frac{\sigma_{1}}{2} V_{1}+\frac{1}{d} \sigma_{2} \sqrt{V_{1}} \sqrt{V_{2}},
\end{aligned}
$$

as a consequence, noting that $\frac{1}{d} \sigma_{2} \sqrt{V_{1}} \sqrt{V_{2}} \leq \frac{\sigma_{1}}{2} V_{1}$ can be written as $\frac{1}{d^{2}} \sigma_{2}^{2} V_{2} \leq \frac{\sigma_{1}^{2}}{4} V_{1}$ :

$$
\left(\frac{\sigma}{d^{2}} V_{2} \leq V_{1}\right) \Rightarrow\left(\dot{V}_{1}(t) \leq-\frac{\sigma_{1}}{2} V_{1}\right)
$$

with $\sigma=4\left(\frac{\sigma_{2}}{\sigma_{1}}\right)^{2}$. That is all we need so far concerning $V_{1}$. Consider $V_{2}$, along solutions to (5) and (10), the following is satisfied (recall that $\left.V_{2}(\tilde{\theta}, P)=\tilde{\theta}^{\mathrm{T}} P^{-1} \tilde{\theta}\right)$ :

$$
\begin{aligned}
\dot{V}_{2}(t) & =-\tilde{\theta}^{\mathrm{T}} P^{-1} \dot{P} P^{-1} \tilde{\theta}+2 \tilde{\theta}^{\mathrm{T}} P^{-1} \dot{\tilde{\theta}} \\
& =-d V_{2}-d \tilde{\theta}^{\mathrm{T}} \Upsilon^{\mathrm{T}} C^{\mathrm{T}} C \Upsilon \tilde{\theta}-2 d \tilde{\theta}^{\mathrm{T}} \Upsilon^{\mathrm{T}} C^{\mathrm{T}} C \eta,
\end{aligned}
$$

since $\tilde{\theta}^{\mathrm{T}} \Upsilon^{\mathrm{T}} C^{\mathrm{T}} C \Upsilon \tilde{\theta} \geq 0, \dot{V}_{2}(t) \leq-d V_{2}-2 d \tilde{\theta}^{\mathrm{T}} \Upsilon^{\mathrm{T}} C^{\mathrm{T}} C \eta$. By invoking the same arguments as after (14) and since $\Upsilon$ is bounded by $M$ according to Lemma 1 , there exists $\gamma \in \mathbb{R}_{>0}$ independent of $d$ such that $\left|2 d \tilde{\theta}^{\mathrm{T}} \Upsilon^{\mathrm{T}} C^{\mathrm{T}} C \eta\right| \leq d \gamma \sqrt{V_{1}} \sqrt{V_{2}}$ so that:

$$
\begin{aligned}
\dot{V}_{2}(t) & \leq-d V_{2}+\gamma d \sqrt{V_{1}} \sqrt{V_{2}} \\
& =-\frac{d}{2} V_{2}-\frac{d}{2} V_{2}+\gamma d \sqrt{V_{1}} \sqrt{V_{2}}
\end{aligned}
$$

since $\gamma d \sqrt{V_{1}} \sqrt{V_{2}} \leq \frac{d}{2} V_{2}$ is equivalent to $\gamma^{2} V_{1} \leq \frac{1}{4} V_{2}$, we deduce that:

$$
\left(\bar{\sigma} V_{1} \leq V_{2}\right) \Rightarrow\left(\dot{V}_{2}(t) \leq-\frac{d}{2} V_{2}\right),
$$

with $\bar{\sigma}=4 \gamma^{2}$. Following the proof of Theorem 3.1 in [9], we are going to define a Lyapunov function for the system (10) based on $V_{1}$ and $V_{2}$. Let $d$ be sufficiently large such that $\frac{\sigma}{d^{2}}<\bar{\sigma}^{-1}$ (i.e. such that the small gain condition is satisifed) and take $\chi>0$ such that:

$$
\frac{\sigma}{d^{2}}<\chi<\bar{\sigma}^{-1} .
$$

We introduce the candidate Lyapunov function, $V=$ $\max \left\{V_{1}, \chi V_{2}\right\}$. Function $V$ is the maximum of two continuously differentiable functions, therefore it is locally Lipschitz and so differentiable almost everywhere according to Rademacher's theorem. Along solutions to (10), when $V=V_{1}$ that means $V_{1} \geq \chi V_{2} \geq \frac{\sigma}{d^{2}} V_{2}$. (15) shows us that $\dot{V}(t) \leq-\frac{\sigma_{1}}{2} V(t)$ almost everywhere. Similarly, when $V=$ $\chi V_{2}$ that means $V_{2} \geq \chi^{-1} V_{1} \geq \bar{\sigma} V_{1}$ so (16) shows us that $\dot{V}(t) \leq-\frac{d}{2} \chi V(t)$ almost everywhere. As a consequence, we have that, almost everywhere: $\dot{V}(t) \leq-\min \left\{\frac{\sigma_{1}}{2}, \frac{d}{2} \chi\right\} V$. Taking $d \geq d^{\star} \geq 1$,

$$
\dot{V}(t) \leq-\min \left\{\frac{\sigma_{1}}{2}, \frac{d}{2} \chi\right\} V \leq-\min \left\{\frac{\sigma_{1}}{2}, \frac{1}{2} \chi\right\} V=:-\lambda V .
$$

Using the comparison principle (see Lemma 3.4 in [10]) and the fact that $V$ is positive definite and radially unbounded, we obtain:

$$
|(\eta(t), \tilde{\theta}(t))| \leq \bar{\beta}(|(\eta(0), \tilde{\theta}(0))|, t) \quad \forall t \geq 0
$$

where $\bar{\beta} \in \mathcal{K} \mathcal{L}$. By the definition of $\tilde{x}$,

$$
\begin{aligned}
|(\tilde{x}, \tilde{\theta})| & =\left|\left(\Delta^{-1} \eta+\Delta^{-1} \Upsilon \tilde{\theta}, \tilde{\theta}\right)\right| \\
& \leq d|(\eta+\Upsilon \tilde{\theta}, \tilde{\theta})| \leq d|\eta+\Upsilon \tilde{\theta}|+d|\tilde{\theta}| \\
& \leq d|\eta|+d(1+M)|\tilde{\theta}| \leq d(1+M)|(\eta, \tilde{\theta})| .
\end{aligned}
$$

From (18) and $\eta(0)=\Delta \tilde{x}(0)-\Upsilon(0) \tilde{\theta}(0)$. As $\Upsilon(0)=0$ by initialisation, we have that $\eta(0)=\Delta \tilde{x}(0)$. Recalling that $|\Delta| \leq 1$ (since $d \geq 1$ ), hence $|\eta(0)| \leq|\Delta||\tilde{x}(0)| \leq|\tilde{x}(0)|$ and we obtain:

$$
\begin{aligned}
|(\tilde{x}(t), \tilde{\theta}(t))| & \leq d(1+M) \bar{\beta}(|(\eta(0), \tilde{\theta}(0))|, t) \\
& \leq d(1+M) \bar{\beta}(|(\tilde{x}(0), \tilde{\theta}(0))|, t) .
\end{aligned}
$$

Therefore, we obtain the desired property (8) with $\beta_{d}(s, t)=d(1+M) \bar{\beta}(s, t)$.

\section{REFERENCES}

[1] G. Besançon, Q. Zhang, and H. Hammouri. High-gain observer based state and parameter estimation in nonlinear systems. In Proceedings of the 6th IFAC Symposium, 2004.

[2] M. Chong, R. Postoyan, D. Nešić, L. Kuhlmann, and A. Varsavsky. Estimating the unmeasured membrane potential of neuronal populations from the eeg using a class of deterministic nonlinear filters. Journal of Neural Engineering, 9:026001, 2012.

[3] M.S. Chong, R. Postoyan, D. Nešić, L. Kuhlmann, and A. Varsavsky. A circle criterion observer for estimating the unmeasured membrane potential of neuronal populations. In Proceedings of the Australian Control Conference, 2011.

[4] F.H. Lopes da Silva, A. Hoek, H. Smith, and L.H. Zetterberg. Model of brain rhythmic activity. Cybernetic, 15:27-37, 1974.

[5] G. Deco, V. Jirsa, P.A. Robinson, M. Breakspear, and K. Friston. The dynamic brain: From spiking neurons to neural masses and cortical fields. Cerebral Cortex, 4(8):1-35, 2008.

[6] M. Farza, M. M'Saad, T. Maatoug, and M. Kamoun. Adaptive observers for nonlinearly parameterized class of nonlinear systems. Automatica, 45:2292-2299, 2009.

[7] P.A. Ioannou and J. Sun. Robust Adaptive Control. Prentice-Hall, New Jersey, 1996.

[8] B.H. Jansen and V.G. Rit. Electroencephalogram and visual evoked potential generation in a mathematical model of coupled cortical columns. Biological Cybernetics, 73:357-366, 1995.

[9] Z.-P. Jiang, I.M.Y. Mareels, and Y. Wang. A Lyapunov formulation of the nonlinear small-gain theorem for interconnected ISS systems. Automatica, 32(8):1211-1215, 1996.

[10] H.K. Khalil. Nonlinear Systems. Prentice-Hall, Englewood Cliffs, New Jersey, U.S.A., 3rd edition, 2002.

[11] S.J. Schiff. Neural Control Engineering: The Emerging Intersection Between Control Theory and Neuroscience. Computational Neuroscience. The MIT Press, 2011.

[12] I.Y. Tyukin, E. Steur, H. Nijmeijer, and C. van Leeuwen. Adaptive observers and parametric identification for systems in non-canonical adaptive observer form. arXiv: 0903.2361, 2009.

[13] F. Wendling, J.J. Bellanger, F. Bartolomei, and P. Chauvel. Relevance of nonlinear lumped-parameter models in the analysis of depth-EEG epileptic signals. Biological Cybernetics, 83:367-378, 2000.

[14] Q. Zhang. Adaptive observer for multi-input-multi-output (MIMO) linear time-varying systems. IEEE Transactions on Automatic Control, 47(3):525-529, 2002.

[15] Q. Zhang. Revisiting different adaptive observers through a unified formulation. In CDC (IEEE Conference on Decision \& Control and European Conference on Control, pages 3067-3072, Seville, Spain, 2005.

[16] Q. Zhang and A. Clavel. Adaptive observer with exponential forgetting factor for linear time varying systems. In CDC (IEEE Conference on Decision and Control) Orlando, U.S.A., pages 3886-3891, 2001. 